\title{
A Centralized Framework for Smart Access Point Selection based on the Fittingness Factor
}

\author{
A. Raschellà, F. Bouhafs, M. Seyedebrahimi, M. Mackay, Q. Shi \\ Department of Computer Science \\ Liverpool John Moores University \\ Liverpool, United Kingdom \\ e-maill: [A.Raschella, F.Bouhafs, M.Seyedebrahimi, M.I.Mackay, Q.Shi]@ljmu.ac.uk
}

\begin{abstract}
This paper focuses on addressing the Access Point (AP) selection problem by relying on a centralized controller that provides a global view of the network. This approach follows the Software-Defined Networking (SDN) concept, which has long been considered in the literature as an innovative method to control management functionalities for wired networks and that is also now becoming a hot topic in the context of Wi-Fi networks. The proposed AP selection approach is based on a novel algorithm which relies on the Fittingness Factor (FF) concept, to maximize a function that reflects the suitability of the available spectrum resources to the application requirements. Specifically, this paper describes the development of a framework that implements the FF-based algorithm for smart AP selection in a centralized controller. The simulated performance comparison of this algorithm against a strategy that maximizes the achievable data rate considered in many papers in the literature, illustrates the important achievements that have been obtained in terms of saved bandwidth and users' satisfaction.
\end{abstract}

Keywords-Access point selection; Fittingness factor; Software Defined Network.

\section{INTRODUCTION}

The overwhelming proliferation of innovative online services and wireless technologies in recent years has resulted in a considerable increase in the use of portable devices, such as smart-phones and tablets. Most of these devices utilise WiFi Access Points (APs), in addition to $3 \mathrm{G} / 4 \mathrm{G}$, to connect to the Internet due to its speed and efficiency. In this context, a challenging problem is how to choose the best AP for a certain user. This paper focuses on the optimal provisioning to the users with the opportunity to join a Wi-Fi AP, not only according to the Received Signal Strength (RSS) under the assumption that APs with a stronger signal will offer better performance, but also by taking into consideration other application specific parameters and Quality of Service (QoS) metrics.

AP selection in Wi-Fi networks has been an interesting topic of research since 2002, when it was observed that considering only the RSS to establish which AP a user should join leads to different problems in the efficiency of the spectrum resources [1]. In fact, although RSS represents a simple method to select an AP, it can lead to imbalanced loads between APs, inefficient rate selection and choice of APs with poor throughput, delay and other performance metrics.
There are many approaches in the literature that proposed AP selection strategies, with the aim to maximize the QoS of a particular Wi-Fi user. In [2], the authors propose an association metric called Estimated aVailable bAnd-width (EVA) that allows them to associate a Wi-Fi user with the AP that provides the maximum achievable throughput. Other contributions consider distributed AP selection strategies relying on the best throughput under frameworks based on game theory. For instance, the approach proposed in [3] aims to maximise the achievable throughput for a Wi-Fi user depending on the number of users associated with the same AP and the data rates these users can achieve. In [4], the authors investigate the AP selection problem with variable channel-width WLANs using an evolutionary game theoretical approach. The authors assumed that all the stations adopt the most efficient Modulation/demodulation and Coding Schemes (MCSs) to achieve their highest bandwidth efficiencies under the power constraints.

In [5], the author formulates the AP selection problem as a non-cooperative game where each user tries to maximize its utility function, defined as the throughput reward minus the fee charged by the AP. In [6], the authors formulate the AP selection problem as a game where players are mobile wireless users who choose radio APs to connect to the network. The authors define a new parameter called Access Point Selection Parameter (APSP) based on the Signal to Interference plus Noise Ratio (SINR). In [7], the authors propose a decentralized scheme that considers a neural network where the mobile devices are able to select the AP that is expected to yield the best throughput according to past experienced performance.

However, the decentralised approach adopted in these contributions does not scale well to the level required in large enterprise Wi-Fi networks, where APs serve a multitude of client devices including smart-phones, laptops, and tablets, each with different levels of requirements. In addition to throughput, optimising the performance of large $\mathrm{Wi}-\mathrm{Fi}$ networks requires addressing other issues related to seamless mobility, loadbalancing, interference management, and AP selection. Such challenges can only realistically be addressed if a centralised management approach is adopted.

Software-Defined Networking (SDN) [8] has emerged as an efficient and flexible network management approach for large networks. By decoupling the control plane from the data plane,

This work has received funding from the European Union's Horizon 2020 Research and Innovation programme under Grant Agreement no. 644262 as part of the Wi-5 project. 
SDN can centralise the network management operations in a single entity, often referred to as a Controller. This centralised management approach allows us to programme large networks through the OpenFlow protocol [9].

More recently, researchers started to consider SDN as a more efficient approach to address the challenge of managing wireless networks, including Wi-Fi [10]. Projects such as OpenSDWN [11], EmPOWER [12], and Odin [13] developed Wi-Fi network management frameworks based on SDN. Their main contribution is the extension of SDN network programming API to support Wi-Fi management operations, such as Service Set IDentifier (SSID) management, client associations, and radio configuration. In this context, we propose a novel AP selection approach based on the SDN concept, where the controller selects the best AP using a smart AP selection algorithm based on an innovative Fittingness Factor (FF) concept.

The novel contributions of this paper include:

- Design and assessment of an innovative smart algorithm for AP selection, which relies on the FF concept. In detail, the FF inherently considers the heterogeneity of the requirements for the different stations accessing the network, so that not all the APs are equally appropriate for all the users depending on the application needs. Moreover, as we explain throughout this paper, the algorithm has been designed in order to provide satisfactory performance to both an individual user and the overall Wi-Fi network. A performance evaluation through simulations that compare the algorithm proposed in this paper to another approach considered in the literature will be provided;

- Development of a framework that implements the FFbased smart algorithm in a centralized controller through a MATLAB-based simulator. Thus, the proposed framework will be able to capture the multiuser perspective in a centralized way, hence providing a single decision-making point. This solution can be exploited in crowded enterprise Wi-Fi networks, avoiding the inter-terminal information exchange required in other collaborative decentralized approaches such as the ones proposed in [2]-[7].

The rest of the paper is organized as follows: in Section II we present the SDN-based Wi-Fi management framework, and the assumptions made while designing the proposed AP selection approach. In Section III, we formulate the AP selection problem, and describe our smart AP selection algorithm based on the FF. In Section IV we present the simulation model we used to evaluate our algorithm. Evaluation results are presented in Section V. Finally, Section VI points out concluding remarks and future works.

\section{SDN-BASED WI-FI MANAGEMENT FRAMEWORK}

In our work, we assume that a large Wi-Fi network consists of $N$ APs, controlled by an SDN Controller as illustrated in Fig. 1. In SDN, the management algorithms are implemented as applications on top of the controller, which are triggered according to network conditions.

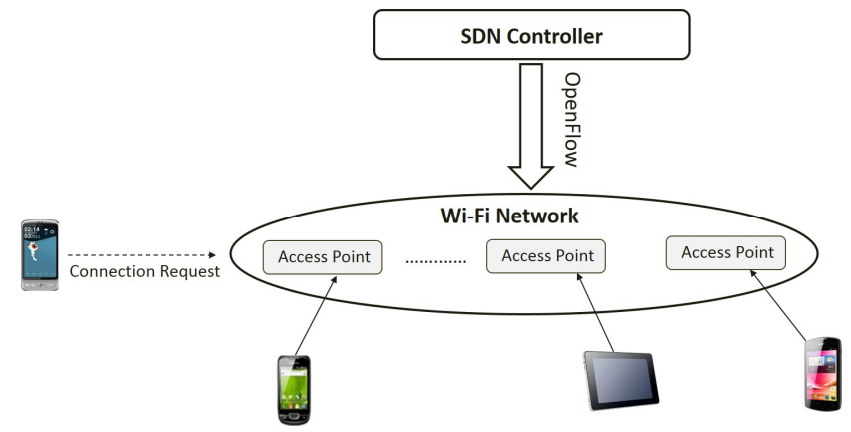

Fig. 1. SDN-Based Wi-Fi Network Management

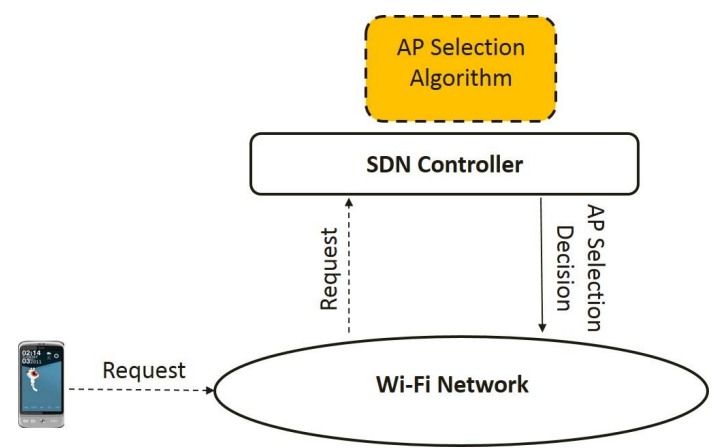

Fig. 2. AP Selection Approach Using SDN Concept

The controller executes the control application and applies the configuration resulting from this execution on the managed network. In the context of the AP selection approach proposed in our work, the SDN controller, upon receiving a terminal connection request redirected by the Wi-Fi network, triggers the AP selection application as illustrated in Fig. 2.

The AP selection application is based on a smart algorithm which assigns a FF to each AP according to the service it can provide to the wireless terminal requesting connection. This algorithm is supported by two functionalities implemented in the Controller: Provided quality assessment and Required quality assessment. The Provided quality assessment gives information on the bit-rate that an AP can achieve when serving the terminal requesting connection. This assessment is achieved by computing the link capacity in terms of the bit rate, which in turns depends on the channel bandwidth assigned to each AP and the monitored inter-AP interference within the network.

The Required quality assessment translates the QoS requirements of the terminal requesting connection into a bitrate metric. The QoS requirements of the terminal depend on the nature of the data flow that the terminal is sending and receiving. These QoS requirements are either proactively programmed into the SDN controller [14], or reactively inferred through QoS detection techniques such as Machine Learning (ML) [15]. In this work we assume that the information used by this functionality to detect the QoS requirements is available. As a reference, the ML-based classification approach proposed and assessed in [15] can be adopted to detect the traffic, and then the QoS requirements can be easily implemented to work in the framework of this paper. 


\section{FITTINGNESS FACTOR BASED AP SELECTION ALGORITHM}

Focusing now on the smart algorithm implemented in the controller, the considered FF consists of a metric between 0 and 1 according to the suitablity of an AP to serve a new user's data flow. The suitablity of an AP is determined using the data bit rate required by the new flow and the bit rate that the AP can achieve. The FF considered in this approach is based on the function formulated in [16]. The developed smart algorithm also makes use of a standard deviation factor of the FFs computed in each available AP in the network. This standard deviation factor ensures that the performance of the overall network is maintained as much as possible when an AP starts serving a new flow. The FF metric for an AP $n$, which serves a flow $j$, is given by the following formula:

$$
f_{j, n}=\frac{1-e^{-\frac{\Gamma \cdot U_{j, n}}{\rho \cdot\left(R_{j, n} / R_{r e q, j}\right)}}}{\lambda}
$$

Here, $R_{\text {req, } j}$ is the bit rate required for flow $j$ and supposed to be obtained using the Required quality assignment functionality. $R_{j, n}$ denotes the bit rate served by AP $n$ when serving flow $j$ and supposed to be computed using the Provided quality assessment functionality. $U_{j, n}$ is the utility function used to depict the QoS perceived by the users in a wireless network [17]. In this context, the function refers to the bit rate achievable by the user $j$ from the AP $n$ with the requested bit rate, and it is defined by the following formula:

$$
U_{j, n}=\frac{\left[\rho \cdot\left(R_{j, n} / R_{r e q, j}\right)\right]^{\xi}}{1+\left[\rho \cdot\left(R_{j, n} / R_{r e q, j}\right)\right]^{\xi}}
$$

$\lambda$ is a normalization factor given by:

$$
\lambda=1-e^{-\frac{\Gamma}{(\xi-1)^{1 / \xi}+(\xi-1)^{(1-\xi) / \xi}}}
$$

$\Gamma, \xi$ and $\rho$ are parameters that reflect the degree of suitability between the requirements and the APs, provided by the FF through (1). Specifically, considering for instance $\Gamma=1, \xi=5$ and $\rho=1.3$ in (1)-(3), the FF for the flow $j$ in AP $n$ reaches its maximum value when the available bit rate $R_{j, n}$ equals the required bit rate $R_{\text {req.j. }}$. The standard deviation of the FFs can then be computed in each accessible AP. The formulation of the standard deviation includes both the FFs of all the previous flows active in the AP $n$ and $f_{j, n}$ computed using (1), and is given by:

$$
\sigma_{j, n}=\sqrt{\frac{\sum_{k=1}^{K}\left(f_{k, n}-\overline{f_{n}}\right)^{2}}{K}} \text { where } \overline{f_{n}}=\frac{1}{K} \sum_{k=1}^{K} f_{k, n}
$$

Note that the standard deviation defines the variation in terms of the average FF that serving a new flow causes on each AP. Each time a new user tries to join the network, the smart connectivity algorithm based on the FF selects through (5) the AP represented by the so-called network FF (i.e., net $f_{j}$ ) that optimises the following parameters: (i) The FF metric that identifies the AP that can serve best the flow requirements of the new user; (ii) The standard deviation factor that maintains the performance of the overall network as much as possible. This optimisation problem, formulated in (5), means that the smart connectivity algorithm based on the FF proposed in this paper aims to optimise the individual performance of the AP associated with the new user while trying to safeguard the overall network performance.

$$
n e t_{-} f_{j}=\arg \max _{n \in\{1, \ldots, N\}}\left\{f_{j, n}\left(1-\sigma_{j, n}\right)\right\}
$$

Focusing now on the algorithm implemented in the controller, each time that a new flow joins the network, the controller will execute the smart connectivity algorithm based on the FF through (1)-(5) making use of the quality information obtained from the Provided quality assessment and Required quality assessment functionalities. After the computation of the optimized network FF parameter achieved through (5), if such a value will be above a threshold to be defined, the corresponding new flow will be allocated to the selected AP. Otherwise, the controller will trigger a reassessment of the required quality to the Required quality assessment functionality. Further details on the implementation of the algorithm will be provided in next section.

\section{EVALUATION SCENARIO}

An area of $1050 \times 1050 \mathrm{~m}^{2}$ is considered with 60 APs at a minimum distance of 75 meters and with a transmit power of $25 \mathrm{dBm}$. Moreover, a free space path loss with exponent 2 is considered. In this scenario, we simulated a station trying to connect to the network every 3 minutes from different and random positions within the network area. We also simulated an application on the station trying to receive a flow of data with different bit rate requirements, as summarised in Table I. In this table we introduce a Quality Grade (QG) which reflects the class of the traffic the application is receiving once connected to the Wi-Fi network, with $\mathrm{QG}=1$ meaning that the traffic has the lowest requirement, and $\mathrm{QG}=3$ meaning that the traffic has the highest requirement.

The controller starts by considering the highest requirement $(\mathrm{QG}=3)$ traffic. For a flow $j$, the algorithm computes net $f_{j}$ considering all the APs accessible from the station requesting connection. If net $f_{j} \geq$ net $f_{\text {th }}$ (where net $f_{\text {th }}$ is the selected threshold), the AP $n$ will be associated with the station receiving flow $j$. If no AP satisfying the quality requirement threshold, net $f_{\text {th }}$, is found, the controller downgrades the quality requirement of the flow to the class below it, i.e. $\mathrm{QG}=2$, and repeats the computation process of net $f_{j}$. The process is repeated until either an AP that satisfies the flow requirement is found, or the quality requirement class reaches $\mathrm{QG}=1$. If no $\mathrm{AP}$ that satisfies the requirement threshold when $\mathrm{QG}=1$ is found, the controller will allocate to the flow the AP represented by net $f_{j}$ when $\mathrm{QG}=3$.

TABLE I. TABLE 1 - BIT RATE REQUIREMENTS

\begin{tabular}{|c|c|c|}
\hline Application & QG & Bit rate (Mbps) \\
\hline \multirow{2}{*}{ Video streaming } & 1 & 1.5 \\
\cline { 2 - 3 } & 2 & 3 \\
\cline { 2 - 3 } & 3 & 6 \\
\hline
\end{tabular}


Different values of net $f_{\text {th }}$ have been considered for the assessment of the proposed algorithm during the simulations. Other parameters are $\Gamma=1, \xi=5$ and $\rho=1.3$. The channel selection procedure considered for the APs of the simulated network and coordinated by the controller has been implemented in order to reduce as much as possible the inter interference among the APs, taking into account the following factors: (i) the Wi-Fi system properties (e.g. IEEE 802.11's standard channel characteristics); (ii) the actual network topology (i.e. APs' distribution throughout the network); and (iii) the desired resource management criteria (e.g. the assigned channels, interference related QoS or handover requirements).

In order to evaluate the performance of the smart connectivity functionality, we devised the most relevant AP allocation strategy available in the literature that acts as a comparison candidate. This strategy associates an AP to a station according to the best achievable data rate by each AP, as proposed in [2] and [4]. Moreover, the evaluation of the smart connectivity is based on the following performance metrics: (1) Data Bit Rate: This metric represents the data rate in terms of kbps achieved by each new flow trying to join the network and averaged for all the flows; (2) Wasted Bandwidth: This metric represents the average extra bandwidth served to all the flows which had their bit-rate requirements satisfied; (3) Station Satisfaction: This metric is the ratio of the assigned bit rate by the required one (e.g., the value corresponding to $\mathrm{QG}=3$ in the case of the FF-based algorithm) averaged for all the flows.

\section{PERFormance RESUlts}

Considering the configuration previously described, the FFbased algorithm and the strategy that maximizes the achievable data rate (named data rate-based from now on) have been executed in the controller every time that a new user tried to join the network. In the case of the FF-based algorithm, different thresholds have been considered in the evaluation. In detail, net $f_{\text {th }}$ values are, respectively, 0.4, 0.6 and 0.8.

In Fig. 3, the achieved data rate evolution is represented as a function of the number of the users for both algorithms. This figure shows that the data rate-based algorithm achieves better performance than the FF-based one in terms of the data bit rate for all the considered thresholds. This result could be expected, as the data rate-based algorithm aims to achieve the maximum bit rate. Moreover, from the figure, no significant differences are observed for the considered thresholds. In details, the FFbased algorithm achieves a significant increase of the data bit rate up to approximately 50 flows that have joined the network when selecting net $f_{t h}=0.4$. However, Fig. 3 shows that in a scenario representing a crowded Wi-Fi network (e.g., from approximately 700 connected flows) the threshold of the FFbased algorithm does not affect the performance much in terms of the achieved data rate.

Fig. 4 and Fig. 5 show the performance results in terms of wasted bandwidth and station satisfaction in the case of the FFbased and data rate-based algorithms. Fig. 4 shows that the data rate-based algorithm tends to offer flows more wasted bandwidth than the FF-based algorithm. This means that the
FF-based solution provides an important improvement in terms of saved bandwidth for all the threshold values in comparison to the data rate-based strategy. In fact, no significant differences in terms of wasted bandwidth are observed when considering different thresholds in the FF-based algorithm. Fig. 5 also shows that the bandwidth saved by the FF-based algorithm does not affect the stations satisfaction in the case of thresholds 0.4 and 0.6. In fact, considering these thresholds, the FF-based algorithm offers a better station satisfaction than the data ratebased algorithm. This proves the bandwidth allocation efficiency of the FF-based algorithm. This result is due to the better distribution of the flows obtained by the FF-based algorithm, as it helps in finding and associating the most suitable AP to a flow, which does not necessarly correspond to the one guaranteeing the highest data rate.

Hence, considering these results, a good trade-off between the saved bandwidth and station satisfaction offered by the FFbased algorithm can be the selection of a threshold between 0.4 and 0.6. For instance, when 960 users connect to the network and in the case of net $f_{\text {th }}=0.6$, the FF-based algorithm allowed an improvement of approximately $70 \%$ in terms of the saved bandwidth and $15 \%$ in terms of the station satisfaction, comparing with the data rate-based algorithm.

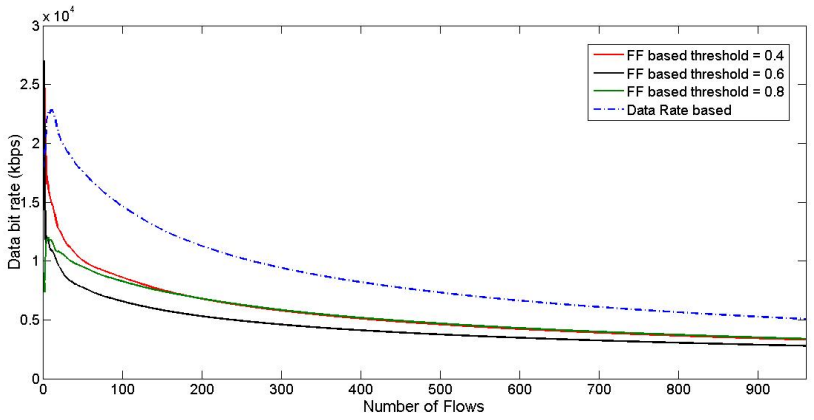

Fig. 3. Data bit rate as a function of the number of flows.

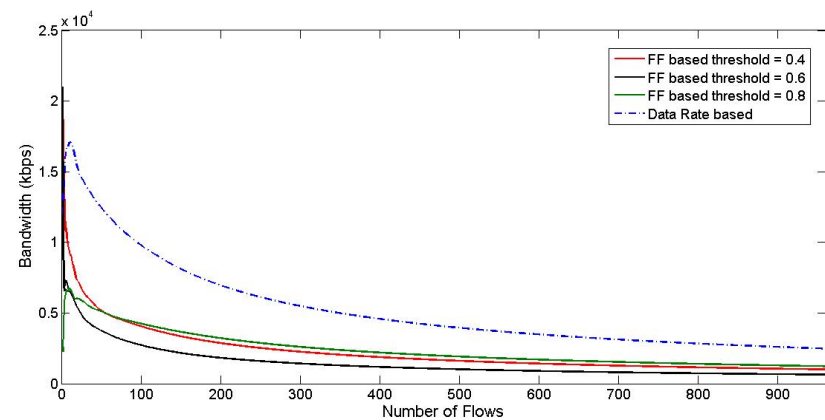

Fig. 4. Wasted Bandwidth as a function of the number of flows.

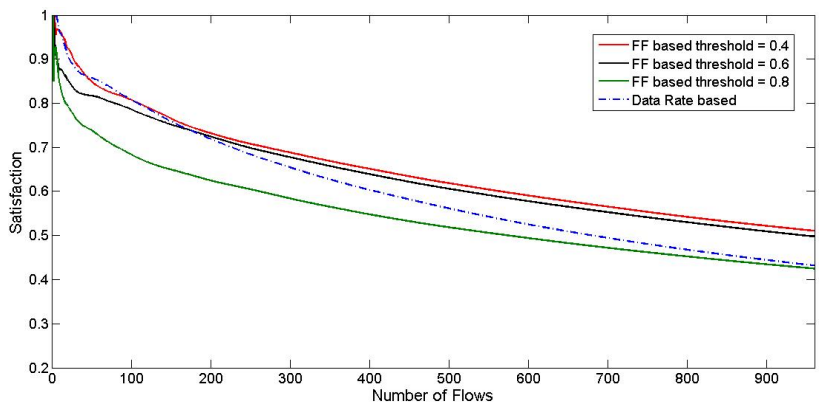

Fig. 5. Satisfaction as a function of the number of flows. 


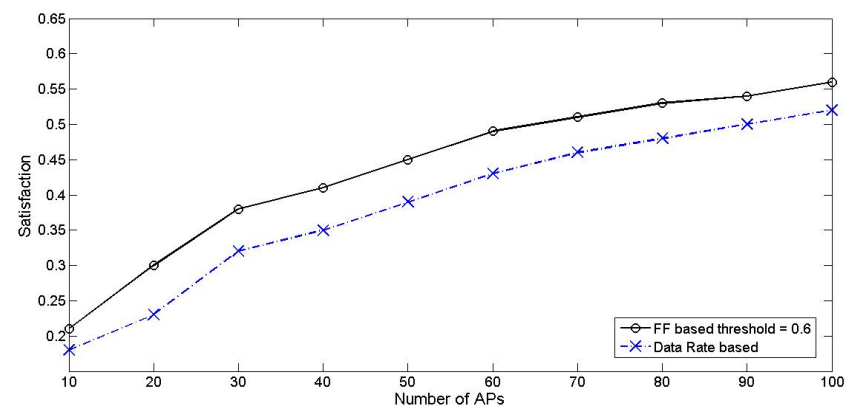

Fig. 6. Averaged Satisfaction as a function of the number of APs.

Finally, considering for instance net $f_{t h}=0.6$, Fig. 6 illustrates the performance results in terms of station satisfaction as a function of the number of available APs in the case of the FF-based and data rate-based algorithms. In this evaluation, a new flow has been generated every 3 minutes in a random position until reaching a number of users equal to 960 . This figure represents the converged value of the station satisfaction averaged for the 960 flows considering different numbers of available APs.

Fig. 6 shows that the performance achieved by both algorithms is an increasing function of the number of APs. In fact, as it would have been expected, the greater the number of APs, the better the performance of both algorithms. However, it is worth mentioning that for all the values of the number of APs, the FF-based algorithm achieves an improvement of approximately $15 \%$ in terms of station satisfaction in comparison with the data rate-based one.

\section{CONCLUSIONS AND FUTURE WORKS}

This paper has presented a centralized framework for AP selection based on the SDN concept, which has emerged as an efficient network management approach for large networks. The proposed framework implements a smart algorithm based on the Fittingness Factor (FF) metric that considers the heterogeneity of the requirements for the different stations accessing the network. To highlight the efficiency of the proposed algorithm, a comparison has been performed against a strategy that maximizes the achievable data rate considered in several papers found in the literature. We have demonstrated that the proposed FF-based algorithm obtains significant improvements in terms of saved bandwidth and users' satisfaction.

Future work will deal with the inclusion in the developed framework of a cross-layer approach based on monitoring procedures that gather information related to the quality of the connection and application-specific parameters, which will support the FF-based algorithm. The proposed algorithm will then be assessed in new scenarios including QoS requirements for different kinds of common online applications. Moreover, the algorithm will be implemented in a real-time testbed in order to assess the benefits achieved through the use of a SDN controller also in a real-time environment.

\section{REFERENCES}

[1] G. Judd, and P. Steenkiste, "Fixing 802.11 access point selection," ACM SIGCOMM Comp. Comm. Review, vol. 32, no. 3, pp. 31, Jul. 2002.

[2] H. Lee, K. Seongkwan, L. Okhwan, C. Sunghyun, and J. Sung, "Available bandwidth based association in IEEE 802.11 Wireless LANs," Int. Symp. on Modeling, analysis and simulation of wireless and mobile systems (MSWiM), Vancouver, Canada, 27-31 Oct. 2008.

[3] Y. Li-Hsing, L. Jia-Jun, and L. Che-Ming, "Stability and Fairness of AP Selection Games in IEEE 802.11 Access Networks", IEEE Transactions on Veh. Tech., vol. 60, no. 3, pp. 1150-1160, Mar. 2011.

[4] X. Chen, W. Yuan, W. Cheng, W. Liu, abd H. Leung, "Access Point Selection under QoS Requirements in Variable Channel-Width WLANs", IEEE Wireless Communications Letters, vol. 2, no. 1, pp. 114-117, Feb. 2013.

[5] L. Chen, “A Distributed Access Point Selection Algorithm Based on Noregret Learning for Wireless Access Networks”, Veh. Tech. Conf. (VTCSpring), Taipei, Taiwan, 16-19 May 2010.

[6] M. Liyanage, J. Chirkova, abd A. Gurtov, "Access Point Selection Game for Mobile Wireless Users", Int. Symp. on a World of Wireless, Mobile and Multimedia Net. (WoWMoM), Sydney, Australia, 16-19 Jun., 2014.

[7] B. Bojovic, N. Baldo, and P. Dini, "A Neural Network Based Cognitive Engine for IEEE 802.11 WLAN Access Point Selection”, Consumer Com. \& Networking Conference (CCNC), Las Vegas, Nevada, USA, 1417 January 2012.

[8] "Software-defined networking: The new norm for networks," Palo Alto, CA, USA, White Paper, Apr. 2012. [Online]. Available: https://www.opennetworking.org/images/stories/downloads/whitepapers/wp-sdnnewnorm.pdf.

[9] N. McKeown, et. al. "OpenFlow: enabling innovation in campus networks”, ACM SIGCOMM Comp. Comm. Review, vol. 38, no, 2, pp. 69-74, 2008.

[10] C. Monsanto, et al., "Composing Software-Defined Networks", USENIX Symposium on Networked Systems Design and Implementation (NSDI), Philadelphia, PA, USA, 19-20 Jun. 2014.

[11] J. Schulz-Zander, et al., "OpenSDWN: programmatic control over home and enterprise WiFi", ACM SIGCOMM Symp. on Software Defined Networking Research, Santa Clara, CA, 14-17 Mar. 2016.

[12] R. Riggio, T. Rasheed, and F. Granelli, "EmPOWER: A Testbed for Network Function Virtualization Research and Experimentation", IEEE SDN for Future Networks and Services (SDN4FNS), Trento, Italy 11-13 Nov. 2013.

[13] J. Schulz-Zander, L. Suresh, N. Sarrar, and A. Feldmann, "Programmatic Orchestration of WiFi Networks," USENIX Symposium on Networked Systems Design and Implementation (NSDI), Philadelphia, PA, USA, 1920 Jun. 2014.

[14] R.Wallner, and R. Cannistra, "An SDN Approach: Quality of Service using Big Switch's Floodlight Open-source Controller", Proceedings of the Asia-Pacific Advanced Network 2013 vol. 35, p. 14-19, 2013.

[15] Nguyen, G. Armitage, P. Branch, and Z. Zander, "Timely and continuous machine-learning-based classification for interactive IP traffic," IEEE/ACM Transactions on Networking, vol. 20, no. 6, pp. 1880-1894, Dec. 2012.

[16] A. Raschellà, J.Pérez-Romero, O. Sallent, and A. Umbert, "On the use of POMDP for Spectrum Selection in Cognitive Radio Networks", Int. Conf on Cognitive Radio Oriented Wireless Networks (CROWNCOM), Washington DC, USA, 8-10 Jul. 2013.

[17] L. Badia, M. Lindstrom, J. Zander, and M. Zorzi, "Demand and pricing effects on the radio resource allocation of multimedia communication systems," Global Comm. Conf. (GLOBECOM 2003), San Francisco CA USA, 1-5 Dec. 2003. 